\title{
Application of protoplast fusion to the nonconventional yeast
}

\author{
Kevin Kavanagh* and Peter A. Whittaker \\ *National Cell and Tissue Culture Centre. Dublin City University, Glasnevin, Dublin 9, Ireland. \\ and 'Department of Biology, St. Patrick's College, Maynooth. Co. Kildare. Ireland
}

\begin{abstract}
Protoplast fusion has made a significant contribution to our understanding of the genetics and biochemistry of the nonconventional yeasts, and it has facilitated the creation of novel strains of yeast that display enhanced biotechnological potential. This article presents an examination of the means of isolating, reverting, and fusing yeast protoplasts, as well as an analysis of the products resulting from such fusions. Although this review is primarily concerned with the impact protoplast fusion has made on our knowledge of the nonconventional yeasts, where appropriate, reference to data gained from work with the conventional yeasts, i.e., Saccharomyces or Schizosaccharomyces, is made.
\end{abstract}

Keywords: Protoplast fusion; cybrid; hybrid: yeast; biotechnology

\section{Protoplast isolation}

$\Lambda$ protoplast may be defined as an osmotically fragile cell completely devoid of cell wall material. Eddy and Williamson ${ }^{1}$ first demonstrated the liberation of protoplasts from Saccharomyces cerevisiae and Saccharomyces carlsbergensis by the action of the enzymes present in Suc d'Helix pomatia. Because of their inherent osmotic fragility, the protoplasts had to be maintained in an environment rendered isotonic by the addition of an usmotic stabilizer. In the absence of the restraining effect of an intact cell wall, a protoplast assumes a spherical shape in an isotonic buffer, to minimize the surface-to-volume ratio. The terms protoplast and spheroplast are often used interchangeably but it should be recognized that the latter term is reserved for osmotically fragile cells enveloped in cell wall material. ${ }^{2}$ As a result of the presence of such wall material, a spheroplast may retain its original cellular shape in isotonic buffer.

Yeast cell wall degradation and subsequent protoplast liberation are most frequently achieved enzymatically, ${ }^{3}$ although mechanical methods have also been employed. ${ }^{4,5} \mathrm{~A}$ wide range of lytic preparations is now available for protoplast isolation from the nonconventional yeasts, although the optimum one for a particular species may have to be

Address reprint requests to Dr. Kavanagh at the National Cell and Tissue Culture Centre. Dublin City University. Glasnevin. Dublin 9. Ireland Received 22 September 1994; revised 24 February 1995; accepted 8 March 1995 determined by pilot experimentation. Novozym 234 (Novo Biolabs, Denmark), originally known as Mutanase, is produced by the submerged fermentation of a strain of Trichoderma harzianum and contains three principal polysaccharases: $\alpha$-1,3-glucanase, $\beta$-1,3-glucanase, and chitinase. It is capable of liberating protoplasts from a range of yeasts ${ }^{6.7}$ and filamentous fungi ${ }^{8,9}$ Funcelase (Yakult Honsha, Tokyo, Japan) is a purified $\beta$-1,3-glucanase preparation from a strain of Trichoderma viride that is efficient in protoplast liberation from a range of yeasts and filamentous fungi. ${ }^{10.11}$ The ability of the gastric juices of the snail $H$. pomatia to degrade yeast cell walls was discovered by Giaja in $1914,{ }^{12}$ although protoplast formation was not observed, because of the absence of an osmotic stabilizer. The mycolytic properties of snail gut juice have been used to liberate protoplasts from a range of yeasts, ${ }^{13}$ and such preparations are available under a variety of brand names: Helicase (Reactifs I.B.F., Garenne, France), Glusulase (Endo Labs, NY, USA), and $\beta$-glucuronidase (Sigma, St. Louis, MO, USA). The active components of snail gut juice preparations include lipase, phospholipase, glucanase, and mannanase. ${ }^{8}$

A number of factors affect the rate of yeast protoplast liberation and subsequent stability. As a culture passes from the exponential to the stationary phase of growth, the cell wall becomes more resistant to enzyme-induced degradation. ${ }^{14}$ Sulphydryl agents have been used extensively with a variety of yeasts in conjunction with lytic preparation(s), to increase the rate of protoplast release from exponential phase cells $s^{6,15}$ or to facilitate the release of protoplasts from 
stationary phase cells. is Sulphydryls are employed for this purpose, because of their ability to reduce the disulphide bonds present in yeast cell walls. Dithiothreitol and $\beta$-mercaptoethanol are the most frequently employed sulphydryl agents. ${ }^{13}$ As a result of their osmotic fragility, protoplasts require an environment rendered isotonic by the presence of an osmotic stabilizer. Sorbitol and mannitol have been the most frequently used osmotic stabilizers for yeast proto plasts, ${ }^{6,7,1.3}$ although inorganic salts such as potassium chloride may also be employed. The viscosity of the protoplast buffer has been shown to affect the rate of protoplast liberation from cells of the yeasts $S$. cerevisiae and Pachusolen tannophilus. ${ }^{17}$

\section{Protoplast regeneration}

Protoplasts of the majority of yeast species are capable of cell wall regeneration, and thereby reestablish the normal cell cycle in medium solidified by the addition of gelatin 18 or agar, ${ }^{19}$ or are thickened by the presence of polyethylene glycol (PEG). ${ }^{20}$ As exceptions, protoplasts of Schizosaccharomyces pombe and Nadsonia elongata are capable of this in osmotically stabilized liquid media. ${ }^{21.22}$ The presence of a barrier around the protoplast, provided by the agar or gelatin, prevents the loss of cell wall components into the surrounding medium, facilitates their accumulation on the surface of the protoplast, and hence allows the formation of the nascent wall. ${ }^{20.22}$ The appearance of mannan (a cell wall component) in the medium during the cultivation of proto plasts of Candida albicans in a liquid environment was attributed to the absence of a barrier capable of restricting matrix component loss. ${ }^{23}$ Devoid of the restraining force of the intact cell wall, protoplasts become spherical in isotonic medium, and because the individual components of the regenerating cell wall are deposited isotropically, the resulting structure will be spherical rather than of the characteristic strain-specific shape. ${ }^{22,24}$ Protoplasts of the xylose fermenting yeast Pachysolen tannophilus were shown to be incapable of wall regeneration and thus reversion, whereas spheroplasts of this yeast were reversion-competent. ${ }^{25}$ It was demonstrated that in this case, remnants of the original cell wall were required for the assembly of the new wall. It was postulated that the wall remnants acted as foci for the deposition of new wall material.

Upon completion of the yeast cell wall, multipolar budding may occur in the first generation, and the usual cellular morphology reappears in the second generation. ${ }^{26}$ Removal of the cell wall during protoplasting uncouples the processes of karyokinesis and cytokinesis, and the relationship resumes only following the completion of a functional cell wall.

\section{Means of inducing protoplast fusion}

Yeast protoplasts may be induced to fuse, under the appropriate physiologic conditions, by electrical or chemical means. The ability to fuse plant protoplasts using an electric field was discovered by Senda, ${ }^{27}$ and the technique was further developed with yeast protoplasts by Zimmermann and co-workers. ${ }^{28-30}$ Electrofusion requires the sequential application of an alternating current $(\mathrm{AC})$ and a direct current (DC) to produce reversible membrane breakdown and concomitant protoplast fusion. Protoplasts in a nonhomogenous AC field display dipoles (protoplast dielectrophoresis), which cause their movement to regions of higher field intensity, where alignment occurs to give rows of protoplasts similar in appearance to chains of pearls. ${ }^{31}$ The $\mathrm{AC}$ field causes lateral diffusion of membrane proteins, leading to the creation of protein-denuded regions on the surfaces of closely apposed protoplasts. ${ }^{32}$ Fusion is achieved by the application of short pulses of the DC current, which produce reversible membrane alteration leading to pore formation, preferentially at the regions of contact of adjacent protoplasts. Resultant changes in permeability and protoplast swelling expand the interprotoplast cytoplasmic continuities to give single spherical structures encompassing the nuclei and cytoplasmic contents of the individual protoplasts.

To date, chemical means - in particular, the use of poly(ethylene glycol) (PEG) - of achieving the fusion of protoplasts of nonconventional yeasts have been the most frequently employed. The fusogenic properties of polymers of ethylene glycol. in combination with calcium. have been well established since they were first identified using plant protoplasts. ${ }^{33.3+}$ PEG has been used to induce the fusion of a variety of cell types including hen erythrocytes, ${ }^{35}$ hen erythrocytes with yeast protoplasts, ${ }^{36}$ and human fibroblasts, ${ }^{37}$ and in the construction of intraspecific, ${ }^{38}$ interspe${\text { cific, }{ }^{39} \text { and intergeneric }}^{40}$ yeast hybrids. The ability of PEG to initiate membrane fusion arises from its capacity to induce the formation of nonspecific cellular aggregates ${ }^{33.36 .41}$ and to produce shrinkage by cellular dehydration. ${ }^{42} \mathrm{Al}-$ though PEG-induced fusion of yeast protoplasts may occur in the absence of calcium, ${ }^{43,44}$ its presence is essential for quantitative results. Intraspecific hybrid production from the PEG-induced fusion of protoplasts of $C$. albicans and $S$. cerevisice is greally increased when calcium propionate, as opposed to the more frequently employed calcium chloride. is the source of the cation. ${ }^{45}$ It was postulated that the propionate anion enhanced the fusion frequency by binding to the etheric oxygen of PEG and potentiating the fusogenicity of the polymer.

The concentration of PEG employed to induce protoplast fusion is critical, because at low levels it is ineffective as an osmoticum, and protoplast swelling occurs, which may ultimately result in lysis. IIigh concentrations of $P E G$ are known to be toxic to yeast protoplasts, producing irreversible hypertonic shrinkage, ${ }^{\text {th }}$ although a certain degree of dehydration is a prerequisite for the fusion process. ${ }^{33}$ The highest fusion frequencies are generally obtained at concentrations giving optimum protoplast survival. ${ }^{+7,48}$ Interbrand and batch variations in the fusogenicity and toxicity of PEG have been observed ${ }^{3 \% .49}$ that may be attributable to the presence of impurities or degradation products. ${ }^{44.50} \mathrm{PEG}$ is susceptible to auto-oxidation initiated by a variety of factors, ${ }^{51}$ and degradation products released by autoclaving have been implicated in reduced yeast protoplast reversion and hybrid yield, ${ }^{52.53}$ lowered hybridoma yields, ${ }^{50}$ and suboptimal yields of heterokaryons from fusions of plant protoplasts. ${ }^{19}$

The fusion of protoplasts of nonconventional yeasts may be achieved by mixing protoplasts of complementary strains 
and incubating in the presence of PEG and the calcium cation. ${ }^{38}$ The protoplast mixture may subsequently be diluted with osmotically stabilized buffer and embedded in osmotically stabilized selection medium. Hybrid selection is usually based on complementing nutritional requirements or the acquisition of resistance to a combination of antibiotics not present in either of the parents. ${ }^{54}$ Plasmogamy may be detected if one of the fusion partners is a petite (or respiratory deficient) strain; mitochondrial transfer is accompanied by the restoration of respiratory competence in the petite strain. ${ }^{55-57}$ Counterselection is achieved by plating the parental petite strain on media containing the nonmetabolizable carbon source, glycerol.

\section{Cybrid formation}

Cytoplasmic coalescence is an inevitable product of the fusion process occurring as a result of the expansion of the fusogen-induced interprotoplast continuities ${ }^{41}$ Cybrids may be defined as fusion products in which the cytoplasmic contents of the fusing protoplasts merge without the concomitant fusion of, or exchange of genetic information between, the nuclei. In fusions of respiratory sufficient (grande) and deficient (petite) strains of $S$. cerevisiae, respiratory competence and nuclear complementation were demonstrated in hybrids that indicated that plasmogamy and karyogamy had been achieved. ${ }^{55}$ Respiratory competence was restored to petite strains of $S$. cerevisiae following PEG-induced fusion with anucleate miniprotoplasts encapsulating functional mitochondria. ${ }^{56}$ The fusion of protoplasts of a mitochondrial mutant strain of Candida utilis with those of a grande strain of $S$. cerevisiae succeeded in restoring respiratory competence. ${ }^{57}$ The resulting fusants possessed functional mitochondria in an orientation, as evident in electronmicrographs, similar to that found in respiratory competent strains of C. utilis. Studies of erythromycin resistance in Kluyveromyces lactis by means of protoplast fusion revealed that in that particular case, the resistance determinant was located on the mitochondrial genome. ${ }^{58}$ Treatment of cells resistant to this antibiotic with ethidium bromide rendered them respiratory-deficient as well as obviating resistance to erythromycin, and because ethidium bromide specifically affects cellular mitochondrial DNA. it was concluded that the resistance in this case was mitochondrially encoded. In Yarrowia lipolytica, protoplast fusion was employed to transfer oligomycin resistance to a sensitive strain without the concomitant occurrence of karyogamy, thus indicating that the resistance factor was cytoplasmically encoded. ${ }^{59}$ Use of the technique described above ${ }^{59}$ may facilitate the investigation of the mitochondrial genetics of this and other yeasts.

In fusions involving protoplasts of respiratory competent strains, the individual mitochondrial genomes may not be retained equally in the hybrids. Using the differential densities of $K$. fragilis and $K$. lactis mitochondrial DNA, the preferential retention of the $K$. fragilis DNA by the hybrids was demonstrated. ${ }^{39}$ Mitochondrial transfer during protoplast fusion occurs at a greater frequency than nuclear fusion in S. cerevisiae ${ }^{60}$ and K. lactis, ${ }^{61}$ indicating that transfer of cytoplasmic organelles between fusing protoplasts is a product of cytoplasmic coalescence, whereas karyogamy proceeds only when the correct physiologic conditions prevail. This indicates that the majority of fused protoplasts in $K$. lactis do not achicve karyogamy and so may be undetectable when nuclear complementation is the sole selection criterion. ${ }^{.1}$

\section{Products of protoplast fusion}

\section{Heterokaryon formation}

Following the induced fusion of protoplasts, the parental nuclei exist temporarily within a common cytoplasm before proceeding, potentially, to karyogamy. Such an heterokaryotic state is analogous to the transient state encountered immediately after cellular fusion during yeast conjugation. ${ }^{62}$ Heterokaryosis is a rare event in yeasts under normal conditions, but frequently occurs in the hyphae of a range of filamentous fungi. Unstable heterokaryotic fusants have been obtained from intraspecific fusions involving strains of C. tropicalis. ${ }^{6.3}$ These could be maintained only by application of selection pressure, the absence of which allowed segregation to the parental genomes. Heterokaryotic nuclei could be induced to fuse, but the products remained unstable and segregated to give various recombinant progeny. Spontaneous karyogamy in heterokaryons of $C$. albicans has been detected ${ }^{6 / 4}$ in which the products either stabilized as tetraploids or randomly lost a number of chromosomes prior to stabilization as aneuploids. A high incidence of heterokaryon formation was noted in protoplast fusions involving nonhybridizing strains of the genus Kluyveromyces. ${ }^{65}$ The instability of these fusants suggests that the cell wall does not constitute the only barrier to genetic exchange between distantly related yeasts, and that some other mechanism of "self-recognition" may govern the interaction of exogenous nuclei. because it was evident that removal of the cell wall did not alter the degree of genetic relatedness between strains or facilitate the formation of stable hybrids.

\section{Genetics of fusion products}

Hybrids which contain unequal contributions of DNA from the parental strains have been frequently obtained as products of protoplast fusion. Hybrids resulting from the intraspecific fusion of strains of $K$. lactis contained a quantity of DNA considerably less than the expected diploid level, and it was suggested that chromosome elimination following protoplast fusion had occurred. ${ }^{66}$ Provost ${ }^{40}$ suggested that the products of the interspecific fusion of auxotrophic strains of $C$. mopicalis and Saccharomycopsis fibuligera consisted of the genome of one parent in association with a few chromosomes of the other, which could be lost relatively easily under certain culture conditions. Based on the segregation patterns and growth characteristics of hybrids produced by the fusion of protoplasts of Sch. pombe and Sch. octosporus. it was concluded that the latter yeast was the dominant contributor to the genetic complement of the fusants. ${ }^{67}$ Prototrophic hybrids resulting from the fusion of protoplasts of two complementary auxotrophic strains of $C$. albicans strongly resembled one of the parents in terms of cell wall proteins and the ability to adhere to exfoliated 
human epithelial cells. ${ }^{68}$ The hybrids were stable aneuploids, and analysis suggested that they consisted of the entire genome of the parent with which they shared most characteristics, together with a chromosome(s) from the other parent. Hybrids resulting from the fusion of protoplasts of $C$. boidinii and C. tropicalis were found to consist of the genome of the former parent with a few chromosomes from the latter. ${ }^{53}$ CHEF electrophoretic karyotype analysis of hybrids resulting from the fusion of protoplasts of $S$. cerevisiae wine and Sake yeasts indicated the presence of the entire wine yeast genome together with a single chromosome from the Sake yeast parent. ${ }^{54}$

Two categories of uninucleate hybrids were obtained from the fusion of protoplasts of C. albicans and C. tropicalis: ${ }^{69}$ One type displayed sugar assimilation and isoenzyme patterns similar to the $C$. albicans parent, whereas the second type presented characteristics of either parent. It was determined that, as in the case described earlier, the hybrids consisted of the nuclear genome of one parent plus a portion of that of the second which might have been integrated into the nucleus of the fusant in a temporary or transient state. A similar situation was evident in hybrids resulting from the fusion of S. cerevisae and S. fermentati, where the products displayed the characteristics of the strain that had contributed most to the particular genome. ${ }^{70}$

Using the technique of whole nuclear DNA-DNA reassociation, it was demonstrated that $S$. diastaticus was the dominant contributor of DNA to intergeneric hybrids formed by the fusion of the former yeast with protoplasts of Hansenula capsulata, $H$. wingei, and C. pseudotropicalis. ${ }^{71}$ The predominance of the $S$. diastaticus genome in the fusants was interpreted as indicating that karyogamy had not occurred, but that the transfer of a single chromosome or chromosomal fragments was the extent of the genetic interaction between the parental nuclei. Hybrids produced by the fusion of protoplasts of $S$. cerevisiae and C. utilis displayed characteristics predominantly of the latter yeast. ${ }^{72}$ Spontancous and induced segregation of the fusants indicated that following plasmogamy, either karyogamy had failed to occur, or that in the event of its having proceeded. the chromosomes supplied by $S$. cerevisiae were preferentially lost in a random fashion during subsequent growth. An example of this was the absence of the $S$. cerevisiae ds-RNA plasmids from the fusants that depended for their continued maintenance on the presence of specific parental chromosomal genes that had possibly been lost from, or were never present in, the hybrids. Meiotic segregants derived from $\mathrm{H} 10$, a stable hybrid from the above fusion displaying the a mating type of $S$. cerevisiae, were crossed with $\alpha$-mating type $S$. cerevisiae strains. The resulting progeny contained a high percentage of heterokaryotic cells, which indicated that after protoplast fusion karyogamy was impaired, but that the transfer of chromosomes between parental nuclei was feasible. ${ }^{7.3}$

Prototrophic, uninucleate, diploid hybrids produced by the fusion of protoplasts of Yarrowia lipolytica and $K$. lactis contained DNA derived principally from the former yeast with as little as $14 \%$ (as calculated by the buoyant densities of nuclear DNA) originating from the $K$. lactis genome. ${ }^{74}$ This may have been the product of either of two events. The nuclei of $Y$. lipolytica in a protoplast may have self-fused to form a diploid which, following plasmogamy, received a chromosome from the $K$. lactis genome. Alternatively, the nuclei of both yeasts could have undergone karyogamy to produce a "diploid" which subsequently lost the chromosomes supplied by the $K$. lactis parent to be replaced by those originating from the Yarrowia genome. It was noted that in fusions between $K$. lactis and $K$. fragilis, chromosome loss from the hybrids had occurred after plasmogamy. ${ }^{39}$ Hybrids produced by the fusion of protoplasts of Pichia stipitis strains were initially unstable but stabilized during subsequent culturing. ${ }^{75}$ Stabilization was a product of ploidy reduction and was evident by the reduced incidence of spontaneous and induced segregation associated with the strains of lower ploidy. A similar situation prevailed with polyploids of $C$. shehatae, which also lost chromosomes prior to stabilization at a lower ploidy. ${ }^{76}$ Hybrids resulting from the intraspecific fusion of protoplasts of $C$. blankii were initially unstable, but stabilized following spontaneous reduction in ploidy during cultivation. ${ }^{77}$

The preferential retention of DNA from one parent may be a product of the fusion protocol employed or the selection procedure used to isolate the hybrids. At the cellular level, the extent of mutagenesis required to induce auxotrophic mutations may produce other undetected mutations, some of which might interfere with the ability of the particular strain to replicate DNA. In fusions bearing different numbers of auxotrophic markers, retention of the genome possessing the least number is favored, as less DNA from the fusion partner is required to achieve complementation. ${ }^{74}$

Chromosomal rearrangements following protoplast fusion in C. maltosa were detected by the occurrence of variations in the mitotic segregation patterns of hybrids after ultraviolet irradiation. ${ }^{78}$ These appeared as differences in the order of the genes and the apparent distance from the centromere, and were explained by the mobilization of transposable DNA sequences during or after protoplast fusion. Hybrids resulting from the fusion of protoplasts of Pachusolen tannophilus and S. cerevisiae resembled the latter parent morphologically and in sugar use, although they displayed the ability to use xylose, which was a characteristic of the P. tannophilus parent. Analysis of the chromosomes of the hybrids by (Field Inversion Gel Electrophoresis) FIGE revealed the presence of a banding pattern intermediate between that of the two parents. ${ }^{74}$

\section{Biotechnologic potential of protoplast fusion}

Elevated ploidy as a result of protoplast fusion may influence a range of yeast metabolic processes. ${ }^{80}$ This technique has been applied, with varying degrees of success, to a number of xylose-fermenting yeasts with the aim of optimizing the efficiency of pentose fermentations. An increase in chromosome number enhanced the rate of production, but not the final yield, of ethanol from xylose by stable polyploids of $C$. shehatae. ${ }^{76}$ The increment in fermentative ability was attributed to the increased gene pool. Polyploids of $P$. famnophilus, constructed by prototrophic selection, ${ }^{81}$ displayed a similar trend. ${ }^{82}$ Elevated ethanol productivity by polyploids of an isogenic S. cerevisiae ploidy series was ascribed to a more efficient fermentation process, per unit of cell mass, in the strains of higher ploidy. ${ }^{83}$ In contrast, uni- 
nucleate prototrophic polyploid strains constructed by the PEG-induced fusion of protoplasts of Pichia stipitis showed no clear correlation with ethanol productivity. ${ }^{75}$ Intraspecific protoplast fusion was employed to increase the ploidy of $C$. blankii for use in a single-cell protein production from hemicellulose hydrolysates. ${ }^{77}$ One isolate, a putative aneuploid, had a mean cell volume three times that of the parental strains. During cultivation, this strain underwent ploidy reduction to yield an isolate (UOVS-PB2) that had a cell volume $43 \%$ greater than the parents and $13 \%$ more DNA. Cell and protein yields from simulated hemicellulose hydrolysate tests revealed that this isolate performed less favorably than the parents. The results of this investigation demonstrate that it is feasible to employ protoplast fusion to produce strains with increased cell volume and DNA content which may have potential in single-cell protein production.

Interspecific and intergeneric yeast protoplast fusion offer the possibility of combining, in a novel way, characteristics associated with the parental strains. The fusion of a strain of $S$. cerevisiae tolerant to high concentrations of ethanol with $K$. fragilis. a yeast capable of lactose fermentation, gave aneuploid and polyploid hybrids. ${ }^{8.4}$ A putative diploid was capable of the efficient fermentation of lactose. producing levels of ethanol $30 \%$ greater than the K. fragilis parental strain. A similar result was obtained when an ethanol-tolerant Sake yeast was fused with the lactosefermenting yeast $K$. lactis, to yield a range of hybrids. ${ }^{85}$ One hybrid. strain PN13, a presumed aneuploid, was a more efficient lactose fermenter than the $K$. lactis parent and, when grown on glucose, displayed alcohol dehydrogenase activity intermediate between that of the parents. Hybrids resulting from the fusion of protoplasts of $S$. diastaticus and the osmotolerant $S$. rouxii contained a high amount of DNA contributed by the former yeast, but enough originating from the latter to give an enhanced usmotolerance. ${ }^{86}$ Certain hybrids resulting from this fusion and their segregants displayed greater dough-raising capabilities than the parental strains. The fusion of protoplasts of $S$. cerevisiae and $Z \mathrm{Y}$ gosaccharomyces fermentati yielded prototrophic progeny presenting characteristics of both parents such as the ability to grow on cellobiose and lactic acid. ${ }^{87}$ The hybrids appeared to be stable under nonselective conditions and to have potential as good fermenters of cellulose hydrolysates. Hybrids resulting from the fusion of $P$. tannophilus and $S$. cerevisiae resembled the latter parent morphologically, but displayed the ability to use the pentose sugar, xylose. ${ }^{79}$ This indicated that using the technique of protoplast fusion the genes for xylose use could be transferred from the Pachysolen parent to the hybrids without grossly altering the characteristics of the resulting progeny which resembled the $S$. cerevisiae parent morphologically, and in terms of sugar assimilation. Such an approach could potentially be used to construct a hybrid capable of the fermentation, as well as the use. of xylose. Hybrids resulting from the fusion of protoplasts of $C$. boidinii (a methanol using yeast) and $C$ tropicalis (an n-alkane using yeast) were capable of using both methanol and $\mathrm{n}$-alkane as sole carbon sources, although the ability to use the latter substrate was lost after prolonged storage ${ }^{5.3}$ The examples cited here demonstrate that it is possible to construct strains by protoplast fusion capable of using or fermenting novel combinations of substrates, and that these strains may have industrial potential.

In addition to promoting increased ethanol productivity or enabling the efficient fermentation of novel substrates (or combinations of substrates), protoplast fusion also offers the possibility of enhancing amino acid or enzyme production. Methionine overproduction was introduced into an S. cerevisiae strain bearing six amino acid markers following fusion with protoplasts of S. uvarum resistant to ethionine and capable of secreting L-methionine. ${ }^{88}$ Methionine production and secretion by the hybrids was inferior to that of the $S$. warum parent, and hybrids were still auxotrophic for four requirements, indicating that only partial complementation had occurred. The conversion of starch to ethanol is a multistepped process necessitating the gelatinization, liquefaction, and saccharification of the substrate prior to its fermentation. Fungal glucoamylases are added at the latter stage to produce glucose and maltose sugars. A hybrid strain constructed by the fusion of protoplasts of $S$. diastaticus and S. wrarum could produce and secrete glucoamylase; thus, only half the level of exogenous enzymes was required to achieve the same yield of ethanol from starch ${ }^{89}$

\section{Concluding remarks}

The application of protoplast fusion to the nonconventional yeasts has increased our understanding of many aspects of their biochemistry and genetics and, in some instances, allowed the creation of strains with interesting biotechnologic properties. In the construction of novel strains, protoplast fusion allows the combination of characteristics present in the parents, although the isolation of a strain displaying specific traits may be a matter of good fortune. The advent of more specific means of manipulating genes has meant that protoplast fusion is no longer the principal method for strain improvement or construction. However, it allows the creation of strains embodying a range of characteristics from the parents or displaying polygenic traits that would be difficult to construct using more refined methods.

\section{References}

1. Eddy, A. A. and Williamson. D. H. A method of isolating protoplasts from yeasts. Nature $1957,179,1252-1253$

2. Darling. S. Theilade. J. and Birch-Andersen. A. Kinetic and morphological observations on Saccharomvces cerevisiae during spheroplasi formation. J. Bacteriol. 1969, 98. 797-810

3. Davis, B. Factors influcncing protoplast isolation. In: Fungul Protoplasts: Applications in Biochemistry and Genetics. (Peberdy, J. F. and Ferenczy, L., eds.). Marcel Dekker, New York, 1985, 45-72

4. Necas, O. Regeneration of yeast cells from naked protoplasts. $\mathrm{Na}$ ture 1956, 177, 898-899

5. Villanueva. J. R. and Garcia-Acha, I. Production and use of fungal protoplasts. In: Methods in Microbiology, vol. 4 (Booth. C.. ed.). Academic Press. New York, 1971, 665-718

6. Stephen, E. R. and Nasim. A. Production of protoplasts in different yeasts by Mutanase. Can. J. Microbiol. 1981, 27, 550-553

7. Dickinson. D. P. and Isenberg. I. Preparation of spheroplasts of Schizosacharomyces pombe. J. Gen. Microbiol. 1982, 128, 651654

8. Hamlyn, P. F.. Bradshaw, R. E.. Mellon. F. M.. Santiago, C. M., Wilson. J. M. and Peberdy. J. F. Efficient protoplast isolation from fungi using commercial enzymes. Ensyme Mirroh. Technol. 1981, 3, 321-325

9. Quigley, D. R., Taft, C. S., Stark, T. and Selitrennikoff, C. P. Op- 
timal conditions for the release of protoplasts of Neurospora using Novozym 234. Exp. Mycol. 1987, 11, 236-240

10. Ogawa, K.. Toyama, H. and Toyama, N. Degradation of fungal cell walls and protoplast formation by a mycolytic enzyme produced by Trictoderma viride. Bull. Fac. Agric. Miya-aki Unin. 1979, 26. $387-398$

11. Kavanagh, K. and Whittaker. P. A. Funcelase-an efficient preparation for the isolation of reversion-competent protoplasts from yeasts. Biotechnol. Tech. 1991, 5, 313-316

12. Giaja. J. Sur l'action de quelques ferments sur les hydrates de carbone de la leuvre. Comp. Rendues Seances Soc. Biologie 1914. 77, $2-4$

13. Peberdy, J. F. Mycolytic enzymes. In: Fungal Protoplusts: Applications in Biochemistry and Genetics (Peberdy. J. F. and Ferenczy. L.. eds.). Marcel Dekker, New York, 1985, 31-44

14. Anderson. F. B. and Millbank. J. W. Protoplast formation and yeast cell wall structure: The action of the enzymes of the snail Helix pomatia. Biochem. J. 1966. 99, 682-687

15. Ferenczy, L. Protoplast fusion in yeasts. In: Fungal Protoplasts: Applications in Biochemistry and Genetics (Peberdy, J. F. and Ferenczy, L.. eds.). Marcel Dekker, New York, 1985, 279-305

16. Dowhanick. T. M.. Panchal. C. J. and Stewart, G. G. Effects of pretreatments with sulphydryls and alkylating agents on spheroplast formation from Schwanniomyces species. Cam. J. Microbiol. 1984. 30, 368-374

17. Kavanagh, K. and Whittaker. P. A. Effect of buffer viscosity on the rate of protoplast formation in yeasts. Biomed. Lett. 1991, 5, 313316

18. Necas, $O$. Physical conditions as important factors for the regeneration of naked yeast protoplasts. Nature $1961,184,1664-1665$

19. Svoboda, A. Regeneration of yeast protoplasts in agar gels. Exp. Cell Res. 1966. 44, 640-642

20. Svoboda, A. and Piedra, D. Reversion of yeast protoplasts in media containing polyethylene glycol. J. Gen. Microbiol. 1983. 129, $3371-3377$

21. Necas, O. Svoboda, A. and Havelkova, M. Mechanism of regeneration of yeast protoplasts: Formation of the cell wall in Schizosaccharomves pombe. Folia. Biol. 1968, 14, 80-85

22. Necas, O. Cell wall synthesis in yeast protoplasts. Bacteriol. Rev. 1971. 35, $149 \quad 170$

23. Gopal. P.. Sullivan, P. A. and Shepherd. M. G. Metabolism of $\left[{ }^{14} \mathrm{C}\right]$ glucose by regenerating spheroplasts of Candida albicans. $J$. Gen. Microbiol. 1984, 130, 325-335

24. Svoboda. A. and Necas, O. Regeneration of yeast protoplasts prepared by snail enzyme. Nature 1966, 210, 169-175

25. Kavanagh, K. and Whittaker, P. A. Formation of spheroplasts and protoplasts in the xylose-fermenting yeast Pachysolen tannophilus. Biotech. Appl. Biochem. 1990, 12, 57-62

26. Necas, O. and Svoboda, A. Cell wall regeneration and protoplast reversion. In: Fungal Protoplasts: Applications in Biochemistry and Genetic:s (Peberdy, J. F. and Ferenczy, L., eds.). Marcel Dekker, New York, 1985, 115-1.33

27. Senda, M. Takeda. J.. Abe, S. and Nakamura. T. Induction of cell fusion of plant protoplasts by electrical stimulation. Plant Cell. Physide 1979, 20, 144!-1443

28. Zimmermann. U. and Scheurich. P. High frequency fusion of plant protoplasts by electric fields. Planta 1981, 151, 26-32

29. Halfmann. H. J. Emeis. C. C. and Zimmermann, U. Electro-fusion and genetic analysis of fusion products of haploid and polyploid Saccharmyces yeast cells. FEMS Microbiol. Lett. 1993. 20, 13-16

30. Schnetter, R., Zimmermann, U. and Emeis, C. C. Large scale production of yeast hybrids by electrofusion. FEMS Microbiol. Lett. $1984,24,81-85$

31. Puite. K. J., van Wiksealer. P. and Verhoeven, H. Electrofusion, a simple and reproducible technique in somatic hybridisation of $\mathrm{Nic}$ otiana plumbaginifolia mutants. Plant Cell Rep. 1985, 4, 274-276

32. Hahn-Hagerdal, B、Hosono, K., 7achrisson, A. and Bornman, C. H Polyethylene glycol and electric field treatment of plant protoplasts: Characterisation of some membrane properties. Phvsiol. Plant. $1986.67,359-364$

33. Kao, K. N. and Michayluk, M. R. A method for high frequency intergeneric fusion of plant protoplasts. Planta 1974, 115, 355-367

34. Wallin. A., Glimelius, K. and Erksson. T. The induction of aggre- gation and fusion of Daucus carota protoplasts by polyethylene glycol. Z. Pflanzenphysol. 1974, 74, 64-80

35. Ahkong, Q. F., Fisher, D., Tampion, W. and Lucy. J. A. Mechanisms of cell fusion. Nature 1975, 253, 194-195

36. Ahkong, Q. F., Howell, J. I., Lucy, J. A.. Safwat, F., Davey, M. R. and Cocking, E. C. Fusion of hen erythrocytes with yeast protoplasts induced by polyethylene glycol. Nature $1975.255,66-67$

37. Pontecorvo, G.. Riddle, P. N. and Hales. A. Time and mode of fusion of human fibroblasts treated with polyethylene glycol (PEG). Nature 1977, 265, 257-258

38. Van Solingen, P. and Van der Plaat, J. Fusion of yeast spheroplasts. J. Bacteriol. 1977. 130, 946-947

39. Whittaker, P. A. and Leach, S. M. Interspecific hybrid production between the yeasts Kluveromyces lactis and Kluveromyces fragilis by protoplast fusion. FEMS Microbiol. Lett. 1978. 4, 31-41

40. Provost, A., Bourguignon, C., Fournier, P., Riber. A. M. and Heslot. $\mathrm{H}$. Intergeneric hybridisation in yeasts through protoplast fusion. FEMS Microbiol. Lett. 1978, 3, 309-312

41. Knutton. S. Studies on membrane fusion: fusion of erythrocytes with polyethylene glycol. J. Cell Sci. 1979. 36, 61-72

42. MacDonald, R. I. Membrane fusion due to dehydration by polyethylene glycol. dextran or sucrose. Biochemistr 1985, 24, 4058-4066

43. Curran, B. P. Protoplast fusion in yeast. Ph.D. thesis, University of Dublin. Ireland. 1984

44. Honda, K., Maeda, Y., Sasakawa, S., Ohno, H. and Tsuchida, E. The components contained in polyethylene glycol of commercial grade (PEG-6000) as cell fusogen. Biochem. Biophys. Res. Commune $1981,101,165-171$

45. Kavanagh, K. Walsh. M. and Whittaker, P. A. Enhanced intraspecific protoplast fusion in yeast. FEMS Microbiol. Lett. 1991, 81, 283-286

46. Peberdy, J. F. Fungal protoplasts: 1solation. reversion and fusion. Ann. Rev. Microbiol. 1979, 33, 21-39

47. Ferenczy, L., Kevei, F. and Szegedi. M. Fusion of fungal protoplasts induced by polyethylene glycol. In: Microbial and Plant Protoplasts (Peberdy. J. F.. Rose. A. H., Rogers. H.J. and Cocking. E. C., eds.). Academic Press. London. 1976. 177-187

48. Anne, J, and Peberdy. J. F. Conditions for induced fusion of fungal protoplasts in polyethylene glycol solutions. Arch. Microbiol. 1975. $105,201-205$

49. Chand, P. K.. Davey, M. R., Power. J. B. and Cocking, E. C. An improved procedure for protoplast fusion using polyethylene glycol. J. Plant Physiol. 1988, 133, 480-485

50. Kadisch, J. L. and Wenc, K. M. Contamination of polyethylene glycol with aldehydes: Implications for hybridoma fusion. Hybridoma $1983,2,87-89$

51. Hamburger, R., Azaz, E. and Donbrow, M. Autoxidation of polyoxyethylenic non-ionic surfactants and of polyethylene glycol. Pharm. Acta. Helv: 1981, 50, 10-17

52. Kavanagh, K.. Ghannoum, M., Mansour, I. and Whittaker. P. A. Autoclaved polyethylene glycol decreases yeast protoplast reversion and hybrid yield. Biorechnol. Tech 1990, 4. 281-284

53. Kobori, H.. Takata. Y. and Osumi, M. Interspecific protoplast fusion hetween Cundida tropicalis and Candida boidinii: Characterisation of the fusants. J. Ferment. Biceng. 1991. 72, 439-444

54. Yamazaki, T. and Nonomura, H. Inherent G418-resistance in hybridisation of industrial yeasts. J. Ferment. Biokeng. 1994. 77, 202204

55. Ferenczy. L. and Maraz. A. Transfer of mitochondria by protoplast fusion in Sacharomyces cerevisiae. Nature 1977. 253, 46-47

56. Maraz, A. and Ferenczy, L. Selective transfer of fungal cytoplasmic genetic elements by protoplast fusion. Curr. Microbiol. 1980. 4, 343-345

57. Richard, M. S., Van Broock. M. R. G. and Figueroa. L. I. C. Restoration of respiratory competence in the yeast Candida utilis after somatic fusion with Saccharomvces cerevisiae. Curr. Microbiol. 1987. 16, 109-112

58. Morgan. A. J. Protoplast fusion and the study of mitochondrial genetics in the petite-negative yeast Kluyveromyces lactis. D. Phil. thesis. University of Sussex. England. 1979

59. Matsuoka. M, Uchida, K. and Aiba. S. Cytoplasmic transfer of oligomycin resistance during protoplast fusion of Saccharomycopsis lipolytica. J. Bacteriol. 1982. 152, 530-533.

60. Curran, B. P. and Carter, B. L. A. alpha-Factor enhancement of hy- 
brid formation by protoplast tusion in Succharomyces cerevisiae II Curr. Genet. 1986, 10, 943-945

61. Bugeja, V.C. and Whittaker, P. A. Differential retention of nuclear and mitochondrial markers in protoplast fusion products of Kluyveromyces lactis. Microbios Lelt. $1986,31,69-74$

62. Crandall, M. Mechanisms of fusion in yeast cells. In: Microbiological and Plant Protoplasts (Peberdy, J. F., Ruse. A. H., Rogers, H J. and Cocking. E. C.. eds.). Academic Press, London. 1976. 161175

63. Fournier. P.. Provost. A.. Bourguignon. C. and Heslot, H. Recombination after protoplast fusion in the yeast Candida tropicalis. Arch. Microbiol. 1977. 115, 143-149

64. Sarachek, A., Rhoads. D. D. and Schwarzhoff, R. H. Hybridisation of Candida albicans through fusion of protoplasts. Arch Microbish. 1981, 129, 1-8

65. Johannsen, E., Halland, L. and Operman. A. Protoplast fusion within the genus Kluveromyces van der Walt emend. van der Wall. Cam. J. Microbiol. 1984, 30, 540-552

66. Allmark, B. M. Morgan. A. J. and Whittaker. P. A. The use of protoplast fusion in demonstrating chromosomal and mitochondrial inheritance of respiratory-deficiency in Kluyeromyces lactis, a petit' negative veast. Mol. Gen. Genet. 1978, 159. 297-299

67. Sipic ki, M. Heyer, W. D. and Kohli, J. Preparation and regeneration of protoplasts and spheroplasts for fusion and transformation of Sithisosacharomyces pombe. Curr. Mis robiol. 1985, 12, 169-174

68. Law, C.. Kavanagh. K. and Whittaker. P. A. Analysis of hybrids of Candida albicans formed by protoplast fusion. FEMS Microbiol. Lett. 1994. 115. 77-82

69. Kucsera, J. and Ferenczy, L. Interspecific hybridisation between Candida albicans and Candida tropicalis. FEMS Microbiol. Lett. 1986. 36, 315-318

70. Skala, J., Luty. J. and Kotylak, Z. Interspecific protoplast fusion between the veasts Saccharomices cerevisiae and Sacharomyces: fermenati. Curr. Genet. 1988, 13,101-104

71. Spencer, J. F. I.. Spencer, D. M. Bizeau, C. Vaughan-Martini, A and Martini. A. The use of mitochondrial mutants in hybridisation of industrial yeast strains. V. Relative parental contributions to the genomes of interspecific and intergeneric yeast hybrids obtained by protoplast fusion. as determined by DNA reassociation. Curr. Genet. 1985. 9. 623-625

72. Perez, C.. Vallin, C. and Benitez. J. Hybridisation of Saccharomyes cerretisiae with Candida urilis through protoplast fusion. Curr. Genlet. 1984. 8, 575-580

73. Percz, C, and Benitez. J. Defective karyogamy in meiotic segregants of a Cundida utilis-Saccharmyces cerevisiae hybrid. Curr. Genet. 1986. 10, 639-642

74. Groves. D. P. and Oliver, S. G. Formation of intergeneric hybrids of yeast hy protoplast fusion of Yorrowia and Kluyveromyces species. Citrs. Genet. 1984. 8, 49-55
75. Gupthar, A. S. Construction of a series of Pichia stipitis strains with increased DNA contents. Curr. Gent't. 1987, 12, 605-610

76. Johannsen. E., Eagle, L. and Bredenhann, G. Protoplast fusion used for the construction of presumptive polyploids of the D-xylose fermenting yeast Candida shehatae. Curr. Genet. 1985, 9. 313-319

77. Meyer. P. S., du Preez. J. C.. Wingfield. B. D. and Kilian, S. G. Evaluation of Candida blankii hybrids for hiomass production. J. Biotechnol. 1993, 29, 267-275

78. Klinner, L. and Bottcher, F. Chromosomal rearrangements after protoplast fusion in the yeast Candida maltosa. Curr. Genet. 1985. 9. 619-621

79. Heluane. H.. Spencer, J. F. T.. Spencer. D. de Figueroa, L. and Callieri, D A. S. Characterisation of hybrids ubtained by protoplast fusion between Pachysolen tannophilus and Sactharomves cerevisiale. Appl. Microbiol. Biotechnol. 1993, 40, 98-100

80. Piper, P. W.. Davies. M. W.. Curran. B.. Lockheart. A.. Spalding, A. and Tuite. M. F. The influence of cell ploidy on the thermotolerance of Sacharmyces cerevisiae. Curr. Genet. 1987. 11, 595-598

81. James. A. P. and Zahab. D. M. The construction and genetic anaysis of polyploids and aneuploids of the pentose-fermenting yeast. Puchsolen tumophilus. J. Gen. Microbiol. 1983, 129, 2489-2494

82. Maleszkil. R. James. A. P. and Schneider. H. Ethanol production from various sugars by strains of Pai hysolen tannophilus bearing different numbers of chromosomes I Gen Microhiol. 1983. 129 $249.5-25()(1$

83. Dilorio. A. A., Weathers, P. J. and Camphell. D. A. Comparative enzyme and ethanol production in an isogenic ploidy series. Curr. Genet. 1987, 12, 9-14

84. Farahnak, F.. Seki. T.. Ryu. D. D. R. and Ogrydziak. D. Construction of lactose assimilating and high ethanol producing yeasts by protoplast fusion. Appl. Environ. Microbiol. 1986, 51, 362-367

85. Taya, M., Honda. H. and Kobayashi, T. Lactose-utilising hybrid strain derived from Saccharomyces cerevisiae and Kluvveromvces lac tis by protoplast fusion. Agric. Biol. Chem. 1984. 48, 2239-2243

86. Spencer. J. F. T. Bizeau, C.. Reynolds. N and Spencer. D. M The use of mitochondrial mutants in hybridisation of industrial yeast strains. VI Characterisation of the hybrid. Siccharomyces diastaticus $X$ Saciharomyces rouxii, obtained by protoplast fusion, and its behaviour in simulated dough-raising tests. Curr. Genet. 1985. 9. $649-6.52$

87. Pina. A.. Calderon. I. L. and Benitez, T. Intergeneric hybrids of Sacharmmces cerevisiae and Zrgasacharmmces fermentati obtained by protoplast fusion. Appl. Environ. Microbiol. 1986. 51. $995-1003$

88. Brigidi, P.. Matteuzzi, D. and Fava, F. Use of protoplast fusion to introduce methionine overproduction into Sacharomves cerevisiae. Appl. Microbiol. Biotechnol. 1988. 28, 268-271

89. Russell. I.. Crumplen, C. M., Jones, R. M. and Stewart. G. G. Efficiency of genetically engineered yeast in the production of ethanol trom dextrinised cassava starch. Biotech. Lett. 1986, 8, 169-174 